\title{
AD HOC CONSULTANTS - 2017
}

Adriana Benevides Soares - Universidade do Estado do Rio de Janeiro, Rio de Janeiro-RJ

Adriana Cristina Boulhoça Suehiro - Universidade Federal do Recôncavo da Bahia, Cruz das Almas-BA

Adriana Wagner - Universidade Federal do Rio Grande do Sul, Porto Alegre-RS

Adriano Beiras - Universidade Federal de Santa Catarina, Florianópolis-SC

Adriano Furtado Holanda - Universidade Federal do Paraná, Curitiba-PR

Alessandra Nagamine Bonadio Matta - Universidade Federal de São Paulo, São Paulo-SP

Alessandra Turini Bolsoni-Silva - Universidade Estadual Paulista Júlio de Mesquita Filho, Bauru-SP

Alessandra Turini Bolsoni-Silva - Universidade Estadual Paulista Júlio de Mesquita Filho, Bauru-SP

Alessandro de Oliveira dos Santos - Universidade de São Paulo, São Paulo-SP

Alexandre Dias Porto Chiavegatto Filho - Universidade de São Paulo, São Paulo-SP

Alexsandro Luiz de Andrade - Universidade Federal do Espírito Santo, Vitória-ES

Aline Cristina Martins Gratão - Universidade Federal de São Carlos, São Carlos-SP

Alipio de Sousa Filho - Universidade do Minho, Portugal

Amanda Márcia dos Santos Reinaldo - Universidade Federal de Minas Gerais, Belo Horizonte-MG

Ana Alayde Werba Pichelli - Universidade Federal da Paraíba, João Pessoa-PB

Ana Beatriz Pedriali Guimarães - Pontifícia Universidade Católica do Paraná, Curitiba-PR

Ana Carla Lima Ribeiro-Accioly - Universidade do Estado do Rio de Janeiro, Rio de Janeiro-RJ

Ana Carolina Braz - Universidade Federal de São Carlos, São Carlos-SP

Ana Claudia Moreira Almeida Verdu - Universidade Estadual Paulista Júlio de Mesquita Filho, Bauru-SP

Ana Claudia Souza Vazquez - Universidade Federal do Rio Grande do Sul, Porto Alegre-RS

Ana Cristina Garcia Dias - Universidade Federal de Santa Maria, Santa Maria-RS

Ana Cristina Resende - Universidade Federal de São Paulo, São Paulo-SP

Ana Emília Vita Carvalho - Centro Universitário do Pará, Belém-PA

Ana Flávia de Oliveira Santos - Universidade de São Paulo, Ribeirão Preto-SP

Ana Flávia do Amaral Madureira - Centro Universitário de Brasília, Brasília-DF

Ana Karenina de Melo Arraes Amorim - Universidade Federal do Rio Grande do Norte, Natal-RN

Ana Luiza Gomes Pinto Navas - Faculdade de Ciências Médicas da Santa Casa de São Paulo, São Paulo-SP

Ana Maria Justo - Universidade Federal de Santa Catarina, Florianópolis-SC

Ana Maria Lopes Calvo de Feijoo - Universidade Federal do Ceará, Fortaleza-CE

Ana Maria Pimenta Carvalho - Universidade de São Paulo, Ribeirão Preto-SP

Ana Maria Xavier Faraco - Universidade Federal de Santa Catarina, Florianópolis-SC 
Ana Paula Soares da Silva - Universidade de São Paulo, Ribeirão Preto-SP

Ana Rosa Gliber - Associação de Pais e Amigos dos Excepcionais de Rio Grande Da Serra, Rio Grande da Serra-SP André Luiz Monezi Andrade - Universidade Federal de São Paulo, São Paulo-SP

Andrea Carvalho Beluce - Universidade Estadual de Londrina, Londrina-PR

Andrea Duarte Pesca - Faculdade CESUSC, Florianópolis-SC

Andrea Regina Rosin Pinola - Universidade Federal de São Carlos, São Carlos-SP

Andreia Schmidt - Universidade de São Paulo, Ribeirão Preto-SP

Andrés Pérez-Acosta - Universidad del Rosario, Colômbia

Angela Helena Marin - Universidade do Vale do Rio dos Sinos, São Leopoldo-RS

Ângelo Brandelli Costa - Universidade Federal do Rio Grande do Sul, Porto Alegre-RS

Anna Elisa de Villemor-Amaral - Universidade São Francisco, Campinas-SP

Anselmo Pereira de Lima - Universidade Tecnológica Federal do Paraná, Pato Branco-PR

Antônio dos Santos - Universidade de São Paulo, Ribeirão Preto-SP

Ariana Cosme - Universidade do Porto, Portugal

Ariel César Núñez Rojas - Universidad de Medellín, Colômbia

Aureliano Lopes da Silva Junior - Universidade do Estado do Rio de Janeiro, Rio de Janeiro-RJ

Avelino Luiz Rodrigues - Universidade de São Paulo, São Paulo-SP

Beatriz Oliveira Valeri Pereira da Silva - Universidade Federal do Triângulo Mineiro, Uberaba-MG

Berenice Victor Carneiro - Pontifícia Universidade Católica de Campinas, Campinas-SP

Bruna Larissa Seibel - Universidade Federal do Rio Grande do Sul, Porto Alegre-RS

Bruno Figueiredo Damásio - Universidade Federal do Rio de Janeiro, Rio de Janeiro-RJ

Carina Nunes Bossardi - Universidade Federal de Santa Catarina, Florianópolis-SC

Carla Ferreira de Paula Gebara - Universidade Federal de São Paulo, São Paulo-SP

Carmen Beatriz Fabriani - Centro Universitario das Faculdades Associadas de Ensino, São João da Boa Vista-SP

Carmen Lúcia Cardoso - Universidade de São Paulo, Ribeirão Preto-SP

Carolina Baptista Menezes - Universidade Federal de Santa Catarina, Florianópolis-SC

Carolina Fernandes de Carvalho - Universidade de Lisboa, Portugal

Caroline Tozzi Reppold - Fundação Universidade Federal de Ciências da Saúde de Porto Alegre, Porto Alegre-RS

Cássia Baldini Soares - Universidade de São Paulo, São Paulo-SP

Celina Maria Colino Magalhães - Universidade Federal do Pará, Belém-PA

Ceneide Maria de Oliveira Cerveny - Pontifícia Universidade Católica de São Paulo, São Paulo-SP

Cesar Alexis Galera - Universidade de São Paulo, Ribeirão Preto-SP 
Cintia Bragheto Ferreira - Universidade Federal do Triângulo Mineiro, Uberaba-MG

Claiton Henrique Dotto Bau - Universidade Federal do Rio Grande do Sul, Porto Alegre-RS

Clarissa De Antoni - Universidade Federal de Ciências da Saúde de Porto Alegre, Porto Alegre-RS

Clarissa Mendonça Corradi-Webster - Universidade de São Paulo, Ribeirão Preto-SP

Clarissa Pinto Pizarro de Freitas - Universidade Salgado de Oliveira, Niterói-RJ

Clarisse Pereira Mosmann - Universidade do Vale do Rio dos Sinos, São Leopoldo-RS

Claudia Maria Gaspardo - Universidade de São Paulo, Ribeirão Preto-SP

Claudia Medeiros de Castro - Universidade de São Paulo, São Paulo-SP

Conceição Aparecida Serralha - Universidade Federal do Triângulo Mineiro, Uberaba-MG

Cristian Zanon - Universidade São Francisco, Campinas-SP

Cristina Maria de Souza Brito Dias - Universidade Católica de Pernambuco, Recife-PE

Cristina Cinto Araujo Pedroso - Universidade de São Paulo, Ribeirão Preto-SP

Cristina Maria de Souza Brito Dias - Universidade Católica de Pernambuco, Recife-PE

Cristina Massot Madeira Coelho - Universidade de Brasília, Brasília-DF

Cristine Costa Lacet - Pontifícia Universidade Católica de São Paulo, São Paulo-SP

Dagma Venturini Marques Abramides - Faculdade de Odontologia de Bauru, Bauru-SP

Daniel Bartholomeu - Centro Universitário FIEO, Osasco-SP

Daniel Correa Mograbi - Pontifícia Universidade Católica do Rio de Janeiro, Rio de Janeiro-RJ

Daniela Barsotti Santos - Universidade Federal do Rio Grande, Rio Grande-RS

Daniela Castro Dos Reis - Universidade Federal Rural da Amazônia, Belém-PA

Daniela Dantas Lima - Universidade de Campinas, Campinas-SP

Daniela Fonseca Freitas - Universidade do Porto, Portugal

Daniela Sacramento Zanini - Pontifícia Universidade Católica de Goiás, Goiânia-GO

Danilo Rodrigues Silva - Universidade de Lisboa, Portugal

Débora Dalbosco Dell'Aglio - Universidade Federal do Rio Grande do Sul, Porto Alegre-RS

Denise Dascanio - Universidade Estadual Paulista Júlio de Mesquita Filho, Bauru-SP

Denise De Micheli - Universidade Federal de São Paulo, São Paulo-SP

Denise Falcke - Universidade do Vale do Rio dos Sinos, São Leopoldo-RS

Denise Maria Cogo - Escola Superior de Propaganda e Marketing, São Paulo-SP

Denise Ruschel Bandeira - Universidade Federal do Rio Grande do Sul, Porto Alegre-RS

Diógenes de Souza Bido - Universidade Presbiteriana Mackenzie, São Paulo-SP

Divane de Vargas - Universidade de São Paulo, São Paulo-SP 
Dolores Cristina Gomes Galindo - Universidade Federal de Mato Grosso, Cuiabá-MT

Ederaldo José Lopes - Universidade Federal de Uberlândia, Uberlândia-MG

Edna Lúcia Tinoco Ponciano - Universidade do Estado do Rio de Janeiro, Rio de Janeiro-RJ

Edna Maria Marturano - Universidade de São Paulo, Ribeirão Preto-SP

Edson Massayuki Huziwara - Universidade Federal de São Carlos, São Carlos-SP

Elaine Cristina Gardinal Pizato - Universidade de São Paulo, Ribeirão Preto-SP

Elaine Rabelo Neiva - Universidade de Brasília, Brasília-DF

Elder Cerqueira-Santos - Universidade Federal de Sergipe, Aracaju-SE

Eliana Marcello De Felice - Universidade de São Paulo, São Paulo-SP

Elisa Kern de Castro - Universidade do Vale do Rio dos Sinos, São Leopoldo-RS

Elizabeth Joan Barham - Universidade Federal de São Carlos, São Carlos-SP

Elizabeth Wiese - University College Roosevelt, The Netherlands

Elmir de Almeida - Universidade de São Paulo, Ribeirão Preto-SP

Emerson Diogenes de Medeiros - Universidade Federal do Piauí, Parnaíba-PI

Emerson Fernando Rasera - Universidade Federal de Uberlândia, Uberlândia-MG

Érica Renata de Souza - Universidade Estadual de Campinas, Campinas-SP

Érika Arantes de Oliveira Cardoso - Universidade de São Paulo, Ribeirão Preto-SP

Eucia Beatriz Lopes Petean - Universidade de São Paulo, Ribeirão Preto-SP

Euclides Maurício Trindade Filho - Universidade Estadual de Ciências da Saúde de Alagoas, Maceió-AL

Eva Diniz - Universidade Federal do Rio Grande do Sul, Porto Alegre-RS

Evandro Moraes Peixoto - Pontifícia Universidade Católica de Campinas, Campinas-SP

Evely Boruchovitch - Universidade Estadual de Campinas, Campinas-SP

Evelyn Eisenstein - Universidade do Estado do Rio de Janeiro, Rio de Janeiro-RJ

Everley Rosane Goetz - Universidade Federal de Santa Maria, Santa Maria-RS

Fabiana Freitas - Universidade de São Paulo, Ribeirão Preto-SP

Fabiana Maris Versuti-Stoque - Universidade de São Paulo, Ribeirão Preto-SP

Fabiano Koich Miguel - Universidade São Francisco, Bragança Paulista-SP

Fábio Scorsolini-Comin - Universidade Federal do Triângulo Mineiro, Uberaba-MG

Felipe Bruno Martins Fernandes - Universidade Federal da Bahia, Salvador-BA

Fernanda Aguiar Pizeta - Universidade de São Paulo, Ribeirão Preto-SP

Fernanda Daniel - Instituto Superior Miguel Torga, Portugal

Fernanda Kimie Tavares Mishima Gomes - Universidade de São Paulo, Ribeirão Preto-SP 
Fernando Pessotto - Universidade São Francisco, Campinas-SP

Fernando Santana de Paiva - Universidade Federal de São João Del-Rei, São João Del-Rei-MG

Flávia Cristina Silveira Lemos - Universidade Estadual Paulista Júlio de Mesquita Filho, Assis-SP

Flávia da Silva Ferreira Asbahr - Universidade Estadual Paulista Júlio de Mesquita Filho, Bauru-SP

Flávia de Lima Osório - Universidade de São Paulo, Ribeirão Preto-SP

Flávia Helena Pereira Padovani - Universidade de São Paulo, Ribeirão Preto-SP

Francine Náthalie Ferraresi Rodrigues Queluz - Universidade São Francisco, Campinas-SP

Francisco José Rengifo-Herrera - Universidad de Ibagué, Colômbia

Francois Jacques Tonneau - Universidade Federal do Pará, Belém-PA

Fraulein Vidigal de Paula - Universidade de São Paulo, São Paulo-SP

Geraldo Romanelli - Universidade de São Paulo, Ribeirão Preto-SP

Gimol Benzaquen Perosa - Universidade Estadual Paulista Júlio de Mesquita Filho, Botucatu-SP

Giselle Pianowski - Universidade São Francisco, Campinas-SP

Gláucia Mitsuko Ataka da Rocha - Universidade de São Paulo, São Paulo-SP

Gleiber Couto Santos - Universidade Federal de Goiás, Catalão-GO

Gleici da Silva Castro Perdoná - Universidade de São Paulo, Ribeirão Preto-SP

Gleny Terezinha Duro Guimarães - Pontifícia Universidade Católica do Rio Grande do Sul, Porto Alegre-RS

Gustavo Levandoski - Universidade Federal da Grande Dourados, Dourados-MS

Gustavo Martileli Massola - Universidade de São Paulo, São Paulo-SP

Heloísa Gonçalves Ferreira - Universidade Federal de São Carlos, São Carlos-SP

Hugo Cogo-Moreira - Universidade de São Paulo, São Paulo-SP

Ida Manuela de Freitas Andrade Timóteo Lemos - Universidade de Évora, Portugal

Ione Vasques-Meneses - Universidade de Brasília, Brasília-DF

Isolda Günther - Universidade de Brasília, Brasília-DF

Ivan Sant'Ana Rabelo - Universidade de São Paulo, São Paulo-SP

Ivonise Fernandes da Motta - Universidade de São Paulo, São Paulo-SP

Ivonne Edith Alejo Castañeda - Universidad Católica de Colômbia, Colômbia

Izabel Augusta Hazin Pires Loreto - Universidade Federal do Rio Grande do Norte, Natal-RN

Jaime Barrientos Delgado - Universidad de Santiago de Chile, Chile

Jair Lício Ferreira Santos - Universidade de São Paulo, Ribeirão Preto-SP

Jairo Eduardo Borges-Andrade - Universidade de Brasília, Brasília-DF

Jaqueline Gomes de Jesus - Instituto Federal de Educação, Ciência e Tecnologia do Rio de Janeiro, Rio de Janeiro-RJ 
Jeanny Joana Rodrigues Alves de Santana - Universidade Federal de Uberlândia, Uberlândia-MG

Jefferson de Souza Bernardes - Universidade Federal de Alagoas, Maceio-AL

Jefferson Rodrigues Pereira - Universidade Estácio de Sá, Rio de Janeiro-RJ

Jenny Amanda Ortiz Munõz - Universidade Federal do Rio Grande do Sul, Porto Alegre-RS

João Mazzoncini de Azevedo Marques - Universidade de São Paulo, Ribeirão Preto-SP

Jocemara Ferreira Mognon - Universidade São Francisco, Campinas-SP

Jorge Gato - Universidade do Porto, Portugal

José Aloyseo Bzuneck - Universidade Estadual de Londrina, Londrina-PR

José Carlos Bernardino Carvalho Morgado - Universidade do Minho, Portugal

José Egídio Oliveira - Universidade do Porto, Portugal

José Luis Álvaro Estramiana - Universidad Complutense de Madrid, Espanha

José Miguel Latorre Postigo - Universidad de Castilla-La Mancha, Espanha

Joseane de Souza - Universidade de São Paulo, Ribeirão Preto-SP

Josiane Rosa Campos - Universidade Federal de São Carlos, São Carlos-SP

Juliana Archiza Yamashiro - Universidade Federal de São Carlos, São Carlos-SP

Juliana Barreiros Porto - Universidade de Brasília, Brasília-DF

Juliana de Oliveira Augustin - Universidade Federal de Viçosa, Viçosa-MG

Juliana Vendrúscolo - Universidade de Ribeirão Preto, Ribeirão Preto-SP

Juliane Callegaro Borsa - Pontifícia Universidade Católica do Rio de Janeiro, Rio de Janeiro-RJ

Júlio César Coelho de Rose - Universidade Federal de São Carlos, São Carlos-SP

Júlio Rique Neto - Universidade Federal da Paraíba, João Pessoa-PB

Karina de Melo Conte - Centro Universitário Claretiano de Batatais, Batatais-SP

Katia de Souza Amorim - Universidade de São Paulo, Ribeirão Preto-SP

Katya Luciane de Oliveira - Universidade Estadual de Londrina, Londrina-PR

Lariana Paula Pinto - Instituto de Pós-Graduação e Graduação, Manaus-AM

Laura Vilela e Souza - Universidade de São Paulo, Ribeirão Preto-SP

Leda Mariza Fischer Bernardino - Pontifícia Universidade Católica do Paraná, Curitiba-PR

Leo Lynce Valle de Lacerda - Universidade do Vale do Itajaí, Itajaí-SC

Leonardo Palacios Sánchez - Universidad del Rosario, Colômbia

Liana Fortunato da Costa - Universidade de Brasília, Brasília-DF

Lília Iêda Chaves Cavalcante - Universidade Federal do Pará, Belém-PA

Lirene Finkler - Universidade Federal do Rio Grande do Sul, Porto Alegre-RS 
Luan Flávia Barufi Fernandes - Universidade de São Paulo, Ribeirão Preto-SP

Luana Flavia da Silva Talmelli - Universidade de São Paulo, Ribeirão Preto-SP

Lucas Francisco de Carvalho - Universidade São Francisco, Campinas-SP

Lucia Helena de Freitas Pinho França - Universidade Salgado de Oliveira, Niterói-RJ

Lúcia Vaz de Campos Moreira - Universidade Católica do Salvador, Salvador-BA

Lúcia Yasuko Izumi Nichiata - Universidade de São Paulo, São Paulo-SP

Luciana Carla dos Santos Elias - Universidade de São Paulo, Ribeirão Preto-SP

Luciana de Oliveira Campolina - Centro Universitário de Brasília, Brasília-DF

Luciana Leonetti Correia - Universidade de São Paulo, Ribeirão Preto-SP

Luciana Mourão Cerqueira e Silva - Universidade Salgado de Oliveira, Niterói-RJ

Luciene Alves Miguez Naiff - Universidade Federal Rural do Rio de Janeiro, Seropédica-RJ

Maíra Bonafé Sei - Universidade Estadual de Londrina, Londrina-PR

Makilim Nunes Baptista - Universidade São Francisco, Campinas-SP

Manoel Antônio dos Santos - Universidade de São Paulo, Ribeirão Preto-SP

Marcelo Dalla Vecchia - Universidade Federal de São João Del-Rei, São João Del-Rei-MG

Márcia Denise Pletsch - Universidade Federal Rural do Rio de Janeiro, Rio de Janeiro-RJ

Márcia Helena da Silva Melo Bertolla - Universidade de São Paulo, São Paulo-SP

Marco Aurélio Máximo Prado - Universidade Federal de Minas Gerais, Belo Horizonte-MG

Marcos Gimenes Fernandes - Universidade Estadual de Santa Cruz, Ilhéus-BA

Marcos Hirata Soares - Universidade Estadual de Londrina, Londrina-PR

Margareth da Silva Oliveira - Pontifícia Universidade Católica do Rio Grande do Sul, Porto Alegre-RS

Margarida Pocinho - Escola Superior de Tecnologia da Saúde de Coimbra, Portugal

Maria Beatriz Martins Linhares - Universidade de São Paulo, Ribeirão Preto-SP

Maria Cecilia de Vilhena Moraes Silva - Pontifícia Universidade Católica de São Paulo, São Paulo-SP

Maria Cristina Ferreira - Universidade Salgado de Oliveira, Niterói-RJ

Maria Cristina Quintas Antunes - Universidade de Trás-Os-Montes e Alto Douro, Portugal

Maria Cristina Trigueiro Veloz Teixeira - Universidade Presbiteriana Mackenzie, São Paulo-SP

Maria Leonor Espinosa Eneas - Universidade Presbiteriana Mackenzie, São Paulo-SP

Maria Livia Tourinho Moretto - Universidade de São Paulo, São Paulo-SP

Maria Nivalda de Carvalho-Freitas - Universidade Federal de São João Del-Rei, São João Del-Rei-MG

Maria Odete Simão - Universidade Estadual Paulista Júlio de Mesquita Filho, Botucatu-SP

Maria Pilar Albertin Carbo - Universitat de Girona, Espanha 
Mariana Gonçalves Boeckel - Universidade Federal de Ciências da Saúde de Porto Alegre, Porto Alegre-RS

Mariano Rojas - Universidad Popular Autónoma del Estado de Puebla, México

Marilda Aparecida Dantas - Universidade de Campinas, Campinas-SP

Marília Reginato Gabriel - Universidade Federal do Rio Grande do Sul, Porto Alegre-RS

Marina Cardoso de Oliveira - Universidade Federal do Triângulo Mineiro, Uberaba-MG

Marina Greghi Sticca - Universidade de São Paulo, Ribeirão Preto-SP

Marineia Crosara de Resende - Universidade Federal de Uberlândia, Uberlândia-MG

Marjorie Cristina Rocha da Silva - Universidade São Francisco, Campinas-SP

Marlene Neves Strey - Pontifícia Universidade Católica do Rio Grande do Sul, Porto Alegre-RS

Marta Regina Gonçalves Correia - Universidade Estadual Paulista Júlio de Mesquita Filho, Bauru-SP

Verônica Bender Haydu - Universidade Estadual de Londrina, Londrina-PR

Lupicinio Iñiguez-Rueda - Universitat Autònoma de Barcelona, Espanha

José Augusto Evangelho Hernandez - Pontifícia Universidade Católica do Paraná, Curitiba-PR

Martha Giudice Narvaz - Universidade Estadual do Rio Grande do Sul, Porto Alegre-RS

Martinho Braga Batista e Silva - Universidade do Estado do Rio de Janeiro, Rio de Janeiro-RJ

Marúcia Patta Bardagi - Universidade Federal de Santa Catarina, Florianópolis-SC

Mary Sandra Carlotto - Universidade do Vale do Rio dos Sinos, São Leopoldo-RS

Mauro Luís Vieira - Universidade Federal de Santa Catarina, Florianópolis-SC

Michele Poletto - Faculdade IBGEN, Porto Alegre-RS

Mirella Liberatore Prando - Pontifícia Universidade Católica do Rio Grande do Sul, Porto Alegre-RS

Myriam Moreira Protasio - Universidade Federal Fluminense, Rio de Janeiro-RJ

Naiana Dapieve Patias - Faculdade Meridional, Passo Fundo-RS

Nancy Ramacciotti de Oliveira Monteiro - Universidade Federal de São Paulo, Santos-SP

Narjara Mendes Garcia - Universidade Federal do Rio Grande do Sul, Porto Alegre-RS

Natalia Rejane Salim - Universidade Federal de São Carlos, São Carlos-SP

Nayane Piovezan - Universidade São Francisco, Campinas-SP

Nelson Hauck Filho - Universidade São Francisco, Campinas-SP

Noeli Prestes Padilha Rivas - Universidade de São Paulo, Ribeirão Preto-SP

Normanda Araujo de Morais - Universidade de Fortaleza, Fortaleza-CE

Olga Maria Piazentin Rolim Rodrigues - Universidade Estadual Paulista Júlio de Mesquita Filho, Bauru-SP

Patrícia Alvarenga - Universidade Federal da Bahia, Salvador-BA

Patrícia Porchat Pereira da Silva Knudsen - Universidade Estadual Paulista Júlio de Mesquita Filho, Bauru-SP 
Paula Hayasi Pinho - Universidade de São Paulo, São Paulo-SP

Paula Inez Cunha Gomide - Universidade Tuiuti do Paraná, Curitiba-PR

Paulo Cesar Azevedo Dias - Universidade Católica Portuguesa, Portugal

Pérez Acosta Andrés Manuel - Universidad del Rosario, Colômbia

Pollyanna Santos da Silveira - Universidade Federal de Juiz de Fora, Juiz de Fora-MG

Priscilla Braga Laskoski - Universidade Federal do Rio Grande do Sul, Porto Alegre-RS

Rafael Moura Coelho Pecly Wolter - Université Paris Descartes, França

Randolfo Santos Junior - Faculdade de Medicina de São José do Rio Preto, São José do Rio Preto-SP

Regiane Sbroion de Carvalho - Universidade de São Paulo, Ribeirão Preto-SP

Regina Sonia Gattas Fernandes do Nascimento - Pontifícia Universidade Católica de São Paulo, São Paulo-SP

Renata Da Rocha Campos Franco - Universidade São Francisco, Campinas-SP

Renata Silva de Carvalho Chinelato - Universidade Salgado de Oliveira, Niterói-RJ

Roberta Carvalho Romagnoli - Pontifícia Universidade Católica de Minas Gerais, Belo Horizonte-MG

Rodolfo Augusto Matteo Ambiel - Universidade São Francisco, Campinas-SP

Rodrigo Sanches Peres - Universidade Federal de Uberlândia, Uberlândia-MG

Rommel Melgaço Barbosa - Universidade Federal de Goiás, Goiânia-GO

Ronaldo Pilati - Universidade de Brasília, Brasília-DF

Rosana Suemi Tokumaru - Universidade Federal do Espírito Santo, Vitória-ES

Rosangela Kátia Sanches Mazzorana Ribeiro - Universidade Federal de Mato Grosso, Cuiabá-MT

Rosangela Miola Galvão de Oliveira - Universidade Estadual de Londrina, Londrina-PR

Sandra Adriana Neves Nunes - Universidade Federal do Sul da Bahia, Itabuna-BA

Sebastião de Sousa Almeida - Universidade de São Paulo, Ribeirão Preto-SP

Sérgio Eduardo Silva de Oliveira - Universidade de Brasília, Brasília-DF

Silvana Alba Scortegagna - Universidade de Passo Fundo, Passo Fundo-RS

Sílvia Pereira da Cruz Benetti - Universidade do Vale do Rio dos Sinos, São Leopoldo-RS

Silvia Regina Ricco Lucato Sigolo - Universidade Estadual Paulista Júlio de Mesquita Filho, Araraquara-SP

Silvia Renata Magalhães Lordello Borba Santos - Universidade de Brasília, Brasília-DF

Silvia Terra Ludwig - Pontifícia Universidade Católica do Rio Grande do Sul, Porto Alegre-RS

Simone Agadir de Azevedo Santos - Escola Nacional de Saúde Pública Sergio Arouca, Rio de Janeiro-RJ

Sofia de Oliveira Major - Universidade de Coimbra, Portugal

Soler Esparragoza Franklin Giovanni - Universidad del Rosario, Colômbia

Sonia Alberti - Universidade do Estado do Rio de Janeiro, Rio de Janeiro-RJ 
Sônia Maria Guedes Gondim - Universidade Federal da Bahia, Salvador-BA

Sônia Regina Loureiro - Universidade de São Paulo, Ribeirão Preto-SP

Sonia Regina Pasian - Universidade de São Paulo, Ribeirão Preto-SP

Suely Aldir Messeder - Universidade do Estado da Bahia, Salvador-BA

Susana Coimbra- Universidade do Porto, Portugal

Susana P. Barradas - Universidad de los Andes, Colômbia

Sylvia Domingos Barrera - Universidade de São Paulo, Ribeirão Preto-SP

Tales Vilela Santeiro - Universidade Federal do Triângulo Mineiro, Uberaba-MG

Talita Pereira Dias - Universidade Federal de São Carlos, São Carlos-SP

Tânia Brandão - Universidade do Porto, Portugal

Tatiana Afonso - Universidade Federal do Pará, Belém-PA

Tatiana de Cássia Nakano - Pontifícia Universidade Católica de Campinas, Campinas-SP

Tatiana Pontrelli Mecca - Centro Universitário FIEO, Osasco-SP

Tatiane Paschoal - Universidade Federal de São Carlos, São Carlos-SP

Terezinha Aparecida de Carvalho Amaro - Universidade Presbiteriana Mackenzie, São Paulo-SP

Terezinha Féres-Carneiro - Pontifícia Universidade Católica do Rio de Janeiro, Rio de Janeiro-RJ

Thiago Benedito Livramento Melicio - Universidade do Estado do Rio de Janeiro, Rio de Janeiro-RJ

Valéria Amorim Arantes de Araújo - Universidade de São Paulo, São Paulo-SP

Valéria Marques de Oliveira - Universidade Federal Rural do Rio de Janeiro, Seropédica-RJ

Vanda Lúcia Vitoriano do Nascimento - Pontifícia Universidade Católica de São Paulo, São Paulo-SP

Vanessa Barbosa Romera Leme - Universidade do Estado do Rio de Janeiro, Rio de Janeiro-RJ

Vanessa Cristina Machado - Centro Universitário Barão de Mauá, Ribeirão Preto-SP

Vicente Cassepp-Borges - Universidade Federal Fluminense, Volta Redonda-RJ

Victor Manuel da Conceição Viana - Universidade do Porto, Portugal 